Cahiers de la recherche sur les droits fondamentaux

$2 \mid 2003$

Les titulaires particuliers des droits fondamentaux

\title{
Le juge pénal international, entre droits de la défense et devoirs de justice
}

\section{Grégory Berkovicz}

\section{(2) OpenEdition}

12 Journals

Édition électronique

URL : https://journals.openedition.org/crdf/7737

DOI : $10.4000 /$ crdf. 7737

ISSN : 2264-1246

Éditeur

Presses universitaires de Caen

Édition imprimée

Date de publication : 1 janvier 2003

Pagination : 101-110

ISBN : 2-84133-205-5

ISSN : $1634-8842$

Référence électronique

Grégory Berkovicz, «Le juge pénal international, entre droits de la défense et devoirs de justice», Cahiers de la recherche sur les droits fondamentaux [En ligne], 2 | 2003, mis en ligne le 18 décembre 2020, consulté le 15 novembre 2022. URL : http://journals.openedition.org/crdf/7737 ; DOI : https:// doi.org/10.4000/crdf.7737 


\title{
Le juge pénal international, entre droits de la défense et devoirs de justice
}

\author{
Grégory BERKOVICZ \\ Doctorant en droit public \\ Université de Caen Basse-Normandie
}

I. La consécration des droits de la défense devant le juge pénal international

A. Les sources des droits de la défense

B. La conciliation entre les droits de la défense et le droit des victimes

II. La mise en œuvre des garanties des droits de la défense

A. Les garanties juridictionnelles
B. Les garanties procédurales

Après plus de cinquante ans de léthargie, la Cour pénale internationale a connu un réveil rapide : le 17 juillet 1998, soit quatre années après l'adoption du rapport de la Commission du droit international des Nations Unies ${ }^{1}$, le Statut de Rome est adopté ${ }^{2}$. Le $1^{\text {er }}$ juillet 2002, il entre en vigueur après le dépôt du soixantième instrument de ratification. Du 3 au 10 septembre 2002, la première assemblée réunissant les soixante-dix-huit États parties à la Convention adopte les textes nécessaires au fonctionnement de la future Cour suivant les propositions de la Commission préparatoire qui s'était réunie à New York depuis février $1999^{3}$.

Parmi ces textes, le Règlement de procédure et de preuve $^{4}$ est destiné à compléter les dispositions du Statut relativement à la procédure applicable et à la détermination des peines devant la future juridiction. Ces éléments de fonctionnement s'avéreront d'autant plus importants que la Cour pénale internationale pourra s'appuyer sur son efficience et le haut niveau de respect des droits fondamentaux en matière de standards procéduraux pour fonder la légitimité qu'elle devra acquérir.

En effet, il apparaît, à la lumière de l'expérience des juridictions pénales internationales qui l'ont précédée, que la future Cour ne prouvera sa légitimité et sa crédibilité qu'à travers sa capacité à apparaître comme l'expression de la Justice. Elle n'aura pas le choix entre être " juste et effective » ou être " juste effective », mais elle devra être juste pour être effective ${ }^{5}$.

1. A / 49 / 10, Rapport de la Commission du droit international sur les travaux de sa 46 ${ }^{e}$ session, 2 mai-22 juillet 1994.

2. Le texte du Statut de Rome (A/CONF.183/9), adopté en date du 17 juillet 1998, et amendé par les procès-verbaux en date des 10 novembre 1998 , 12 juillet 1999, 30 novembre 1999, 8 mai 2000, 17 janvier 2001 et 16 janvier 2002, est disponible sur internet: http://www.un.org/law/icc/statute/french/ rome_statute(f).pdf (février 2003).

3. Official Records of the Assembly of States Parties to the Rome Statute of the International Criminal Court (ICC-ASP / 1/ 3 and Corr.1), disponible sur internet: http://www.un.org/law/icc/asp/aspfra.htm (février 2003).

4. Texte final du projet de Règlement de procédure et de preuve, PCNICC / 2000 / INF / 3 / Add.1.

5. L.S. Sunga, "Full respect for the rights of suspect, accused and convict: from Nuremberg and Tokyo to the ICC», in Le Droit pénal à l'épreuve de l'internationalisation, M. Henzelin, R. Roth (dir.), Paris, LGDJ, Genève, Georg, Bruxelles, Bruylant, 2002, p. 217-239: « The ICC will have to learn from 
La nécessité d'un niveau élevé de protection des droits de la défense devant le juge pénal international a été reconnue dès l'établissement des deux tribunaux ad hoc, tout en admettant la spécificité de telles juridictions (I), toutefois la mise en œuvre de ces garanties n'est pas sans soulever certaines interrogations (II).

\section{La consécration des droits de la défense devant le juge pénal international}

Plus que tout autre, en effet, le juge pénal international se doit de porter une attention particulière au respect des droits de la défense. Comme le note Laurence Sinopoli ${ }^{6}$, «la juridiciarisation du conflit ne peut prétendre conduire à son apaisement que si l'institution présente les caractères essentiels de la "justice"; à défaut, elle ne sera qu'un argument supplémentaire de violence».

Cette obligation a été appréhendée dès l'origine lors de la codification des principes de droit international pénal par la Commission du droit international par l'affirmation selon laquelle «toute personne accusée d'un crime de droit international a droit à un procès équitable ${ }^{7}$.

Afin de répondre à cette nécessité, le juge pénal international se réfère aux droits reconnus dans les grands instruments internationaux et régionaux de protection des droits de l'homme (A), qu'il adapte cependant aux spécificités de l'ordre international et de la mission qui lui est dévolue (B).

\section{A. Les sources des droits de la défense}

Les règles procédurales applicables devant les juridictions pénales internationales sont empreintes des instruments universels de protection des droits de l'homme, et notamment du Pacte international relatif aux droits civils et politiques du 16 décembre 1966.

L'article 14 du Pacte prescrit les règles qui constituent le standard minimum en matière de procès équitable et de droits de la défense. Le Pacte reconnaît ainsi le droit de toute personne «à ce que sa cause soit entendue équitablement et publiquement par un tribunal compétent, indé- pendant et impartial, établi par la loi, qui décidera soit du bien-fondé de toute accusation en matière pénale dirigée contre elle, soit des contestations sur ses droits et obligations de caractère civil » (paragraphe 1). Sont ensuite rappelés les principes fondamentaux de procédure pénale: la présomption d'innocence (paragraphe 2), le droit un double degré de juridiction (paragraphe 5), le principe non bis in idem (paragraphe 7). L'article 14 paragraphe 3 prévoit enfin les garanties procédurales essentielles applicables en matière pénale: le droit de l'accusé à être informé de la nature et des motifs des accusations portées contre lui, le droit d'organiser sa défense, le droit à être jugé dans un délai raisonnable, le droit à être présent au procès et à pouvoir assurer sa défense avec l'assistance d'un conseil, le droit d'interroger ou de faire interroger des témoins à décharge, le droit à un interprète et le droit à ne pas être forcé de témoigner contre soi-même.

L'ensemble de ces garanties de caractère universel doit naturellement être respecté par toutes les juridictions pénales internationales ${ }^{8}$. La Chambre d'appel du Tribunal pénal international pour l'ex-Yougoslavie (TPIY) l'a d'ailleurs rappelé dans l'affaire Dusko Tadic (exceptions préjudicielles) ${ }^{9}$.

Dans cette décision, l'accusé, Dusko Tadic, invoquait son droit à être jugé par un tribunal «établi par la loi » et se référait à plusieurs instruments internationaux à caractère régional ou universel ${ }^{10}$. Selon la Chambre, cette objection comporte trois aspects. En premier lieu, le tribunal doit être établi par un acte législatif, ce qui n'est certainement pas le cas eu égard aux spécificités de l'ordre international. En second lieu, cela signifie qu'il a été établi par un organe conformément au droit applicable et sur ce point la Chambre se réfère à la compétence du Conseil de sécurité des Nations Unies au regard du droit international. Enfin, qu'il réponde aux exigences des normes internationales appropriées, ce qui est le cas dès lors que la procédure établie par son Statut et son Règlement de procédure et de preuve est conforme aux standards les plus élevés en matière de protection des droits de l'homme et de garanties juridictionnelles. Audelà de la seule question du respect de certaines garanties par le Tribunal, se pose donc, en écho, celle de sa légitimité juridique et politique ${ }^{11}$.

Les tribunaux pénaux internationaux se réfèrent également aux sources régionales de protection des droits

the mistakes of the ICTY and ICTR to ensure that the suspect, accused and convict enjoy at least the minimum applicable international human rights standards» (p. 218).

6. L. Sinopoli, «Les droits de la défense», in H. Ascensio, E. Decaux et A. Pellet, Droit international pénal, Paris, Pédone, 200o, p. 791-805.

7. «Principes du droit international consacré par le statut du Tribunal militaire international et dans le jugement du tribunal», $\mathrm{V}^{\mathrm{e}}$ principe : «Any person charged with a crime under international law has the right to a fair trial on the facts and law. "Cf. Rapport de la Commission du droit international sur les travaux de sa $2^{e}$ session, 5 juin-29 juillet 1950 (A/1316).

8. Rapport du Secrétaire général des Nations Unies sur la création du TPIY, S / 25704, p. 28, \$106.

9. TPIY, CA, arrêt relatif à l'appel de la défense concernant l'exception préjudicielle d'incompétence, IT-94-1-AR72, 2 octobre 1995, $\$ 42$ : «[le] tribunal doit trouver racine dans une règle de droit et offrir toutes les garanties figurant dans les instruments internationaux pertinents. On peut alors dire que le tribunal est "établi par la loi”."

10. Affaire Tadic (compétence) précitée, IT-94-1-AR72, 2 octobre 1995, $\$ 41$ : article $14 \$ 1$ du Pacte international relatif aux droits civils et politiques, adopté à New York le 16 décembre 1966; article $6 \$ 1$ de la Convention européenne de sauvegarde des droits de l'homme et des libertés fondamentales, adoptée à Rome le 4 novembre 1950 ; article $8 \$ 1$ de la Convention américaine des droits de l'homme, adoptée à San José le 22 novembre 1969.

11. M. Sassoli, «La première décision de la Chambre d’appel du Tribunal pénal international pour l'ex-Yougoslavie: Tadic (compétence)», RGDIP, 1996-1, p. 112-114. 
de l'homme. Ainsi, le TPIY a eu l'occasion d'affirmer le nécessaire respect de certaines garanties prévues par la Convention européenne des droits de l'homme ${ }^{12}$. À cet égard, la force obligatoire des dispositions de la Convention européenne des droits de l'homme vis-à-vis d'une juridiction pénale internationale est loin d'être évidente. Cependant, les États qui coopèrent avec le TTPIY pourraient se voir reprocher, devant la Cour européenne des droits de l'homme, la violation de la Convention par le Tribunal ${ }^{13}$.

L’influence particulière de la Convention européenne des droits de l'homme et de la jurisprudence de la Cour chargée de son application sur l'activité des deux tribunaux pénaux ad hoc est évidente et découle de plusieurs facteurs: la Cour européenne, comme les tribunaux pénaux, est une juridiction internationale et applique à ce titre le droit international, l'inspiration de la Convention est sensible dans les dispositions des statuts des tribunaux et, enfin, la jurisprudence de la Cour offre, étant donné la diversité des traditions juridiques des États qui y sont soumis, un " "échantillon de systèmes juridiques" extrêmement intéressant du point de vue du droit comparé ${ }^{14}$.

L'article $21 \mathrm{du}$ Statut du TPIY ${ }^{15}$ prévoit l'essentiel des droits de l'accusé, à savoir: le principe d'égalité devant la justice, le droit un procès public et équitable, le principe de la présomption d'innocence, ainsi que les principales garanties procédurales invocables devant la juridiction $^{16}$.

Le projet de Statut de la Cour pénale internationale de la Commission pour le droit international des Nations Unies ${ }^{17}$, conformément à ce «modèle», prévoyait de la même manière que les principaux droits de la défense soient mentionnés dans un article unique. Toutefois, au cours des travaux du Comité préparatoire ${ }^{18}$ est apparue la nécessité de renforcer et de détailler davantage les dispositions relatives aux droits de la défense ${ }^{19}$. Dès lors, en lieu et place d'une liste exhaustive de droits, les garanties juridictionnelles et procédurales applicables devant la future Cour pénale internationale sont mentionnées à l'intérieur des dispositions relatives à la procédure. La difficulté d'établir une liste synthétique des principaux droits de la défense s'est trouvée renforcée par l'écart qui existe entre les concepts issus des différents systèmes de droits nationaux. Ainsi, par exemple, les termes de «suspect» et "d'accusé » recouvrent, en fonction des différents systèmes, des significations et des conséquences diverses.

Cependant, il est également apparu que certains droits généraux trouvaient application à différents stades de la procédure. C'est pour cette raison que le Statut définitif comprend un article 66 relatif à la présomption d'innocence et un article 67 intitulé « Droits de l'accusé ». Ce dernier, après avoir rappelé le droit de l'accusé à ce que sa cause soit entendue publiquement, équitablement et de façon impartiale, prévoit une liste de garanties procédurales essentielles. Cette liste des garanties générales est complétée, au fil du Statut, par une série de garanties spécifiques qui résultent de la conciliation nécessaire entre les influences du droit anglo-saxon et du droit continental ${ }^{20}$.

Toutefois, avant d'envisager les garanties apportées aux droits de la défense devant les juridictions pénales internationales, il convient de rappeler que la protection des droits de la défense devant ces juridictions comporte un certain nombre de spécificités qui rendent nécessaire leur adaptation à la nature particulière des personnes et des crimes poursuivis.

12. Affaire Tadic (compétence) précitée, IT-94-1-AR72, 2 octobre 1995 et TPIY, CA, 29 octobre 1997, T. Blaskic, IT-95-14-AR108 bis, $\$ 59$.

13. Hypothèse évoquée notamment lors des débats qui ont suivi l'intervention de X. de Roux, "La Défense devant le Tribunal pénal pour l'exYougoslavie ", in La Justice pénale internationale (Actes du colloque organisé à Limoges les 22 et 23 novembre 2001 dans le cadre des Entretiens d'Aguesseau), S. Gaboriau et H. Pauliat (éd.), Limoges, PULIM, 2002, p. 139.

14. A. Cassese, «L'influence de la CEDH sur l'activité des tribunaux pénaux internationaux», in A. Cassese et M. Delmas-Marty, Crimes internationaux et Juridictions internationales, Paris, PUF, 2002, p. 143-182.

15. Statut du Tribunal international pour juger les personnes présumées responsables de violations graves du droit international humanitaire commises sur le territoire de l'ex-Yougoslavie depuis 1991, Résolution 827 du Conseil de sécurité des Nations Unies du 25 mai 1993, amendé (ci-après : Statut du Tribunal pénal international pour l'ex-Yougoslavie ou TPIY); cf. également l'article 20 du Statut du Tribunal criminel international chargé de juger les personnes présumées responsables d'actes de génocide ou d'autre violations graves du droit international humanitaire commis sur le territoire du Rwanda et les citoyens rwandais présumés responsables de tels actes ou violations commis sur le territoire d'États voisins entre le $1^{\text {er }}$ janvier et le 31 décembre 1994, Résolution 955 du Conseil de sécurité des Nations Unies du 8 novembre 1994 (ci-après: Statut du Tribunal pénal international pour le Rwanda ou TPIR).

16. Article 21, alinéa 4: «Toute personne contre laquelle une accusation est portée en vertu du présent statut a droit, en pleine égalité, au moins aux garanties suivantes: a) à être informée, dans le plus court délai, dans une langue qu'elle comprend et de façon détaillée, de la nature et des motifs de l'accusation portée contre elle; b) à disposer du temps et des facilités nécessaires à la préparation de sa défense et à communiquer avec le conseil de son choix ; c) à être jugée sans retard excessif; d) à être présente au procès et à se défendre elle-même ou à avoir l'assistance d'un défenseur de son choix ; si elle n'a pas de défenseur, à être informée de son droit d'en avoir un, et, chaque fois que l'intérêt de la justice l'exige, à se voir attribuer d'office un défenseur, sans frais, si elle n'a pas les moyens de le rémunérer; e) à interroger ou faire interroger les témoins à charge et à obtenir la comparution et l'interrogatoire des témoins à décharge dans les mêmes conditions que les témoins à charge; f) à se faire assister gratuitement d'un interprète si elle ne comprend pas ou ne parle pas la langue employée à l'audience; g) à ne pas être forcée de témoigner contre elle-même ou de s'avouer coupable.»

17. A / 49 / 10, Rapport de la Commission du droit international sur les travaux de sa $46^{e}$ session, 2 mai-22 juillet 1994.

18. Le Comité préparatoire s'est réuni du 25 mars au 12 avril et du 12 au 30 août 1996 (document A / 51 / 22), puis du 11 au 21 février 1997 (A / AC.249 / 1997 / L.5), du 4 au 15 août 1997 (A / AC.249/1997/L.8/Rev.1), du $1^{\text {er }}$ au 12 décembre 1997 (A/AC.249/1997/L.9/ Rev.1), puis en réunion intersessions à Zutphen, Pays-Bas, du 19 au 30 janvier 1998 (A / AC.249/1998 / L.13) et enfin du 16 mars au 3 avril 1998 (A/ AC.249/1998 / L.11) et adopta son rapport final le 14 avril 1998 (A / CONF.183/2, A / CONF.183 / 2 / Add.1 et A / CONF.183 / 2 / Add.2).

19. H. Friman, «Rights of Persons Suspected or Accused of a Crime», in The International Criminal Court-The Making of the Rome Statute, R.S. Lee (éd.), Londres - Boston, Kluwer Law International, 1999, p. 247-262.

20. Pour une illustration de ces contradictions, cf. infra II / B s'agissant de l'article 63 du Statut de la CPI «Procès en présence de l'accusé». 


\section{B. La conciliation entre les droits de la défense et le droit des victimes}

La place de la victime a été considérée comme largement insuffisante dans les statuts des tribunaux pénaux internationaux ${ }^{21}$. L'article 22 du Statut du TPIY (article $21 \mathrm{du}$ Statut du Tribunal pénal international pour le Rwanda), intitulé «Protection des victimes et des témoins », seule disposition relative aux statuts des victimes, renvoie au Règlement de procédure et de preuve. Ce dernier a cependant prévu un certain nombre de dispositions, reprises dans le Statut de la Cour pénale internationale, relatives à la protection des victimes et prévoyant les garanties essentielles de respect de leur dignité durant le procès pénal international.

Or, les dispositions protectrices des victimes peuvent entrer en conflit avec les garanties de l'accusé du droit à un procès équitable. Il en est ainsi des mesures visant à assurer l'anonymat des victimes ou des témoins, admis notamment dans l'affaire Tadic ${ }^{22}$. La Chambre a considéré que des conditions exceptionnelles, relatives notamment à la permanence d'une situation de conflit armé, pouvaient, à raison des craintes pour la sécurité du témoin et de l'importance du témoignage, justifier l'anonymat des témoins au regard du droit de l'accusé à un procès équitable. Cette jurisprudence prévoit, en conformité d'après certains auteurs ${ }^{23}$ avec les exigences de la Cour européenne des droits de l'homme ${ }^{24}$, certaines limites entourant cette possibilité. Malgré ces précautions, cette position fut largement critiquée, jusque dans les rangs des juges du Tribunal ${ }^{25}$.

À l'inverse, les positions adoptées par certains États vis-à-vis des juridictions pénales internationales sont sous-tendues par l'idée selon laquelle la nature des crimes internationaux et le contexte de leur commission excluent, par nature, le respect des standards procéduraux en matière de droits de la défense. Ainsi, le Rwanda, qui avait pourtant réclamé l'institution d'un Tribunal pénal international, a procédé au jugement par ses tribunaux nationaux d'un grand nombre d'accusés en arguant de l'impossibilité pour le tribunal de prononcer la peine de mort et des lenteurs liées au respect des procédures. De même, on constate que les États-Unis préfèrent procéder par l'intermédiaire d'une justice nationale, militaire et expéditive concernant la poursuite des «terroristes talibans» plutôt que d'en confier le soin à une juridiction créée sous l'égide du Conseil de sécurité des Nations Unies.

À cet égard, il convient de rappeler que tant le Préambule que l'article $1^{\text {er }}$ du Statut de la Cour pénale internationale prévoient que celle-ci exerce sa compétence à l'égard des personnes pour les crimes les plus graves ayant une portée internationale. La compétence de la Cour est donc déterminée par la gravité des infractions qui menacent la paix et la sécurité et touche l'ensemble de la communauté internationale. C'est d'ailleurs sur le fondement de ces dispositions que certains auteurs ${ }^{26}$ contestent l'opportunité de la compétence de la future Cour à l'égard des crimes de guerre, dont la gravité, au regard de la communauté internationale dans son ensemble, peut être variable.

Le choix a finalement été fait, en partie sous l'influence de la France, dans le Statut de la Cour pénale internationale, de conférer aux victimes une place plus éminente que celle qui leur était réservée devant les juridictions pénales internationales ad hoc ${ }^{27}$. Ainsi, l'article 67 précité est suivi immédiatement d'un article 68 consacré à la «Protection et participation au procès des victimes et des témoins » qui prévoit, notamment, l'intervention d'une «Division aux victimes et aux témoins » créée au sein du Greffe de la Cour conformément à l'article 43. En outre, l'article 75, également adopté à l'initiative de la France ${ }^{28}$, prévoit le principe de "Réparation en faveur des victimes ». Dans le cadre de l'étude de l'équilibre entre droit de la défense et droits des victimes, ce dernier article doit être comparé avec son pendant, l'article 85 du Statut, qui prévoit l'«Indemnisation des personnes arrêtées ou condamnées » dans l'hypothèse d'une erreur judiciaire. Enfin, la section III du chapitre IV du Règlement de procédure et de preuve (règles 85 à 99) intitulée «Victimes et témoins » détaille les mesures de protection des victimes et des témoins, les modalités de leur participation aux différentes phases du procès et, notamment, leur représentation, ainsi que les modalités de la réparation en faveur des victimes.

Ces dispositions constituent l'aboutissement de l'évolution de la jurisprudence et du Règlement de procédure et de preuve applicables devant les tribunaux pénaux internationaux, notamment s'agissant des victimes de violences à caractère sexuel et des victimes ou des témoins nécessitant une protection particulière ${ }^{29}$. Elles renforcent la participation des victimes à la procédure, quasiment inexistante devant les tribunaux pénaux ${ }^{30}$.

21. R. Maison, «La place de la victime», in H. Ascensio, E. Decaux et A. Pellet, Droit international pénal, p. 779-784 et, particulièrement, p. 780 , $\$ 4$.

22. TPIY, IT-94-1-T, 10 août 1995, $\$ 53$.

23. R. Maison, «La place de la victime», p. 779-784.

24. CEDH, Kostovsi c. Pays-Bas, 20 novembre 1989, A, n 166 et CEDH, Windisch c. Autriche, 27 septembre 1990, A, $\mathrm{n}^{\circ} 186$.

25. TPIY, IT-94-1-T, 10 août 1995, opinion dissidente du juge Stephen.

26. C. Santulli, «Qu'est-ce qu'une juridiction internationale? Des organes répressifs internationaux à l'ORD », AFDI, 2001, p. 58.

27. F. Harhoff, «The Role of the Parties before International Criminal Courts in Lights of the International Criminal Tribunal for Rwanda », in International and National Prosecution of Crimes under International Law, H. Fischer, C. Kreß et S. R. Lüder (éd.), Berlin, Berlin Verlag, 2001, p. 649.

28. C. Muttukumaru, «Reparation to Victims », in The International Criminal Court..., p. 262-270.

29. O. Sidem-Poulain, «Une consolidation des acquis pour les droits des femmes», FIDH, $\mathrm{n}^{\circ}$ 266, novembre 1998, Rapport de position $n^{\circ} 3:$ analyse du Statut de la CPI, disponible sur internet: http://www.fidh.imaginet.fr (novembre 1998).

30. Pour une analyse synthétique des droits des victimes devant les tribunaux pénaux internationaux et devant la CPI, cf. United Nations, The United Nations and the Development of International Law, 1990-1999, chap. 1, p. 15 et p. 26, disponible sur internet: www.un.org (février 2003). 
Au-delà de la conciliation entre les droits de l'accusé et ceux des victimes, une tension peut apparaître entre le droit pénal international et les objectifs de protection des droits de l'homme. Ainsi, comme le relève William A. Schabas, «il y a souvent incompatibilité (appréhendée) entre l'impératif de la lutte contre l'impunité, dont le but est de promouvoir et de protéger les droits de l'homme, et les garanties procédurales offertes à l'accusé ${ }^{31}$. Il en est ainsi, d'après l'auteur, de la conception très large adoptée par les juridictions pénales internationales de l'élément psychologique de l'infraction. La lutte contre l'impunité semble pouvoir parfois rendre acceptable la condamnation d'une personne moralement innocente ou dont le niveau de culpabilité ne dépasse pas celui de la simple négligence.

Cependant, comme le reconnaît l'auteur, la reconnaissance devant les juridictions pénales internationales de la culpabilité du subalterne qui aura commis un crime en application d'un ordre manifestement illégal ou sous la contrainte, de même que celle du supérieur hiérarchique qui «savait, ou, en raison des circonstances, aurait dû savoir ${ }^{32}$ que ses subalternes commettaient ou allaient commettre un tel crime, a pour objectif de déconnecter la chaîne de commandement devant aboutir à la commission de l'infraction.

De même, la manière dont la question de la proportion entre la peine prononcée et l'infraction commise et celle de la finalité de la peine-punition et exemplarité mais aussi amendement et réhabilitation - ont été à peine effleurées dans le Statut de Rome et dans le Règlement de procédure et de preuve, démontre la difficile conciliation entre la répression des atteintes aux droits de l'homme et le respect de ces droits dans le cadre de cette répression.

Ainsi, l'article 77 du Statut de la Cour prévoit-il le prononcé d'une peine d'emprisonnement à temps de 30 ans au plus et le prononcé d'une peine d'emprisonnement à perpétuité «si l'extrême gravité du crime et la situation personnelle du condamné le justifient », auxquelles peuvent s'ajouter des condamnations pécuniaires. L'article 78 , relatif à la fixation de la peine, n'apporte guère plus de précision et renvoie à la gravité du crime et à la situation personnelle du condamné. Les premiers commentateurs avaient espéré que le Règlement de Procédure et de preuve viendrait combler cette indétermination, toutefois la règle 145 dudit Règlement n'apporte que peu de précisions, tenant notamment aux circonstances atténuantes et aggravantes susceptibles d'être retenues par la Cour.

Si l'on peut comprendre, dans un contexte de conflit armé, la position de certains États qui considèrent, en invoquant le respect des victimes, que des crimes d'une certaine gravité peuvent justifier des mesures exceptionnelles, on ne saurait cependant l'admettre. C'est en effet l'idée même que l'on se fait de la justice et de la paix dans la société internationale qui est en jeu. On renverra sur ce point au raisonnement qui a présidé tant à la création des tribunaux internationaux de Nuremberg et de Tokyo qu'à celle des juridictions internationales contemporaines. C'est ce qui justifie l'attention qui doit être apportée à la mise en œuvre par le juge pénal international des garanties juridictionnelles et procédurales des droits de la défense.

\section{La mise en œuvre des garanties des droits de la défense}

La réponse formulée par les juges du Tribunal militaire international de Nuremberg sur le respect du principe de la légalité des délits et des peines était fondée sur le pouvoir appartenant à chaque État de sanctionner le droit par la création de juridictions spéciales. Cette réponse n'a bien sûr pas été reprise par les tribunaux pénaux internationaux résultant de la volonté du Conseil de sécurité des Nations Unies agissant sur la base du chapitre VII de la Charte des Nations Unies.

En effet, le raisonnement qui sous-tend la justification de la légalité et de la légitimité de ces juridictions pénales internationales s'appuie sur la notion de communauté internationale. Se pose alors la question du lien juridique ainsi établi entre l'individu soumis au jugement et la communauté internationale qui a institué la juridiction. En premier lieu, l'État de nationalité, dont l'individu poursuivi est ressortissant, est réputé avoir accepté la juridiction, que celle-ci résulte d'une convention (CPI) ou d'une décision du Conseil de sécurité sur la base du chapitre VII. Cependant, certains auteurs relèvent que la justification essentielle de la légalité d'une telle juridiction repose sur la garantie du respect des droits de la défense de la personne poursuivie ${ }^{33}$.

Objet de contestations persistantes et juridiction en quête de légitimité donc, le juge pénal international se doit d'opérer un délicat dosage entre le respect des droits de l'accusé et celui de la nécessité de rendre la justice, s'agissant aussi bien des garanties juridictionnelles (A) que des garanties procédurales (B).

\section{A. Les garanties juridictionnelles}

Les garanties juridictionnelles offertes par des juridictions de l'ordre international peuvent sur ce point apparaître plus effectives que celles offertes par des tribunaux internes dont l'impartialité pourrait être contestée. Cette soustraction, par la communauté internationale, de l'accusé à son «juge naturel » pour l'attraire devant une juridiction internationale a été présentée par la Chambre d'appel dans l'affaire Tadic précitée ${ }^{34}$ comme

31. W.A. Schabas, «Droit pénal international et droit international des droits de l'homme: faux frères? », in Le Droit pénal à l'épreuve de l'internationalisation, p. 172.

32. Statut de la Cour pénale internationale, article 28.

33. L. Sinopoli, «Les droits de la défense », p. 797.

34. TPIY, IT-94-1-T, 10 août 1995. 
constituant une garantie d'être jugé de façon plus équitable, plus indépendante et plus désintéressée des faits de la cause que ne pourrait l'assurer une juridiction nationale.

Cette garantie d'indépendance et d'impartialité constitue une exigence forte de la Cour européenne des droits de l'homme conformément à l'article $6 \$ 1$ de la Convention $^{35}$. Or, si d'après certains auteurs cette indépendance est «absolue ${ }^{36}$ s'agissant de la future Cour pénale, elle apparaît selon d'autres observateurs comme beaucoup plus contestable s'agissant du TPIY ${ }^{37}$. À cet égard, il apparaît que certaines difficultés sémantiques et fonctionnelles relevées concernant le TPIY ont été largement revues et corrigées dans le Statut de la future Cour, notamment par l'introduction d'une Chambre préliminaire qui intervient dans la phase préalable au procès et qui est, notamment, chargée d'actes d'instruction sur demande du procureur et de la confirmation des charges sur lesquelles celui-ci entend se fonder. En revanche, les interrogations relatives à l'indépendance « politique » du juge pénal international ne sont, quant à elles, pas levées dans le Statut de Rome. À cet égard, le plus important n'est sans doute pas d'établir le rôle politique du juge pénal international dans le contexte des relations internationales. Le plus important est l'apparence de son rôle politique. Sur ce point, Rony Brauman relevait que "cette justice internationale dans le monde est perçue comme une justice des puissants ${ }^{38}$.

Une autre incertitude a été par ailleurs soulevée concernant les garanties juridictionnelles offertes par les juridictions pénales internationales : celle de leur qualification même de juridictions ${ }^{39}$. Définissant la juridiction comme « un organe qui met fin à un différend par une décision obligatoire rendue en application du droit», le professeur Carlo Santulli en déduit que ni les tribunaux pénaux internationaux, ni la future Cour pénale internationale ne réunissent les trois conditions (différend, application du droit et caractère obligatoire). L'auteur considère, en premier lieu, que dès lors que le TPIY rend des décisions sur la base du code pénal yougoslave, cela a pour conséquence que les décisions rendues ne le sont pas sur la base du droit international. Il considère, en second lieu, que la force obligatoire des décisions rendues repose non sur l'autorité de la chose jugée mais sur la légalité d'exception instituée par le Conseil de sécurité sur la base du chapitre VII. L'argumentation peut paraître sophiste, elle n'est cependant pas dénuée d'intérêt.
Elle revient cependant à contester la capacité effective du Conseil de sécurité à créer une « juridiction».

Le raisonnement de l'auteur concernant la Cour pénale internationale est différent. Il rappelle en effet la nécessité absolue de l'existence d'un différend « au cœur du procès, y compris pénal ». Il poursuit son analyse en rappelant les conditions de la compétence de la Cour qui ne peut, sauf intervention du Conseil de sécurité sur la base du chapitre VII (ce qui renvoie à des conditions analogues à celles des tribunaux ad hoc), connaître une affaire que si l'État de nationalité de l'accusé ou l'État sur le territoire duquel le crime a été commis a conféré juridiction à la Cour. Il en déduit un certain nombre de problèmes juridiques relatifs à l'application du principe nullum crimen, nulla poena sine lege et de celui de l'effet relatif des traités (res inter alios acta). Il en déduit surtout, quelle que soit l'hypothèse, que «le différend supposé entre l'organisation, représentée par le procureur, et le particulier se solde par des obligations pour des tiers au procès, qui n'était pas parties à un différend ». Il précise que, " par le Statut, les États se sont engagés à infliger des punitions aux auteurs de certains faits définis par le traité. La Cour a pour fonction d'indiquer quels sont ces individus dans certains cas, notamment lorsque les personnes soupçonnées par le procureur international ne sont pas punies par les autorités nationales. Un État est alors désigné pour mettre en œuvre la punition ». Il considère donc que la Cour pénale internationale est un organe administratif d'assistance à l'exécution. Cette seconde analyse soulève une interrogation plus fondamentale encore que la précédente, celle de la place respective des individus et des États dans la société internationale.

Le principe général de droit pénal de légalité des délits et des peines évoqué précédemment a fait lui aussi débat, s'agissant de son application par le juge pénal international. Ce principe, rappelé avec force et constance dans les grands instruments universels de protection des droits de l'homme ${ }^{40}$, constitue également une garantie fondamentale conformément à l'article 7 de la Convention européenne des droits de l'homme et à la jurisprudence de la Cour $^{41}$. Or, si d'après certains auteurs ${ }^{42}$, les doutes quant au respect de ce principe par le juge pénal international ne sont qu'apparents, d'autres observateurs soulèvent des objections qui méritent d'être mentionnées. Le débat ne porte plus, comme ce fut le cas au sujet des procès de Nuremberg et de Tokyo,

35. CEDH, 9 juin 1998, Incal c. Turquie, Rec. 1998-IV; E. Decaux et P. Tavernier, «Chronique de jurisprudence de la Cour européenne des droits de l'homme (année 1998)", JDI, 1999, p. 247-248.

36. F. Rubio et S. Moghaddassi, «Les garanties juridictionnelles devant la Cour pénale internationale», Les Petites Affiches, $\mathrm{n}^{\circ}$ 170, 27 août $2001, \mathrm{p} .4-14$.

37. X. de Roux, «La Défense devant le Tribunal pénal pour l’ex-Yougoslavie», p. 133.

38. R. Brauman, « Regards sur le TPIY», in La Justice pénale internationale, p. 146.

39. C. Santulli, «Qu'est-ce qu'une juridiction internationale?...».

40. L'article 11 / 2 de la Déclaration universelle des droits de l'homme et l'article 15 du Pacte relatif aux droits civils et politiques disposent tous deux que «Nul ne sera condamné pour des actions ou omissions qui, au moment où elles ont été commises, ne constituaient pas un acte délictueux d'après le droit national et international. De même, il ne sera infligé aucune peine plus forte que celle qui était applicable au moment où l'acte délictueux a été commis».

41. CEDH, 9 février 1995, Welch c. Royaume-Uni, A, n 307-A; CEDH, C.R. c. Royaume-Uni, A, n 335-C; J.-P. Costa, «Principe de non-rétroactivité des peines et Convention européenne des droits de l'homme", AJDA, 1996, p. 445.

42. Y. Jurovics, «Le procès international pénal face au temps», Rev. sc. crim., octobre-décembre 2001, p. 781-797. 
sur le caractère rétroactif ou non des incriminations portées à l'encontre des accusés.

C'est sur la légalité des peines que se portent les interrogations. S'agissant du TPIY, en premier lieu, il convient de rappeler que sa compétence ratione temporis couvre une période antérieure à sa création. C'est pourquoi l'article 24 du Statut prévoit que le Tribunal «a recours à la grille générale des peines d'emprisonnement appliquées par les tribunaux de l'ex-Yougoslavie». Or, on relève que le Code pénal yougoslave en vigueur au moment des faits prévoyait de sanctionner le crime de génocide et les crimes contre l'humanité par la peine de mort ou, à défaut, par une peine de 20 ans d'emprisonnement. Pourtant, les juges rédacteurs du Règlement de procédure et de preuve ont prévu à l'article 101 que «Toute personne reconnue coupable par le Tribunal est passible de l'emprisonnement pouvant aller jusqu'à l'emprisonnement à vie ${ }^{43}$. S'agissant, en second lieu, de la CPI, il n'est nul besoin d'un tel raisonnement. Ainsi qu'il a été exposé ${ }^{44}$, les juges de la future Cour n'auront à leur disposition pour fixer les peines qu'ils devront prononcer qu'un Statut laconique à peine complété par un Règlement elliptique. En effet, la règle 145 se contente de préciser, pour l'ensemble des infractions punissables prévues au Statut, le principe de l'individualisation du prononcé des peines et de définir sommairement les notions de circonstances atténuantes et aggravantes. Ce dernier point est sans nul doute le plus préoccupant s'agissant de la difficulté à laquelle les futurs juges devront faire face : la grande liberté qui sera la leur dans la fixation des peines entre $a b$ initio en contradiction avec le principe susmentionné.

Dernier point soulevé quant aux garanties juridictionnelles offertes par les juridictions pénales internationales concernant les droits de défense, la question de la détermination précise des règles procédurales garantissant le respect des droits de défense renvoie à la notion de «tribunal établi par la loi » ${ }^{45}$. En effet, s'agissant des tribunaux internationaux de Nuremberg de Tokyo et plus récemment des tribunaux pénaux internationaux de La Haye, l'absence de législateur international a conduit à investir les juges eux-mêmes du pouvoir d'établir et de modifier les règles de procédure applicable. C'est ainsi que le Règlement de procédure et de preuve applicable devant les tribunaux pénaux internationaux ad hoc a déjà fait l'objet d'un grand nombre de modifications qui ont engendré une insécurité juridique et pratique largement contestée tant par la doctrine que par les avocats appelés à plaider devant ces juridictions ${ }^{46}$. Les rédacteurs du Statut de la Cour pénale internationale ont d'ailleurs éviter cet écueil pour l'avenir puisque le Règlement de procédure et de preuve est adopté et modifié par l'Assemblée des États parties.

\section{B. Les garanties procédurales}

La question du jugement par contumace devant les tribunaux pénaux internationaux n'était pas totalement réglée par la rédaction des dispositions de leurs statuts. En effet, l'article 21 du Statut du TPIY prévoit le droit de l'accusé à être présent au procès. Cependant, ce droit aurait pu être interprété comme une faculté, ce qui aurait permis au Tribunal de juger des personnes dûment informées de l'acte d'accusation établir la rencontre, même en leur absence. Il aurait suffi que soient vérifiées les conditions de renonciation à comparaître et de prévoir la faculté de «purger» la contumace. Toutefois, le Tribunal de Nuremberg ayant admis très explicitement et exercé la faculté du jugement par contumace et ayant fait l'objet de critiques vigoureuses sur ce point, la possibilité de juger in absentia est généralement rejetée par la doctrine anglo-saxonne.

Les juges des tribunaux pénaux internationaux contemporains, auteurs du Règlement de procédure et de preuve, imprégnés des exigences procédurales de la Common Law, ont opté pour une procédure excluant la possibilité du jugement par contumace. Toutefois, ils ont prévu une solution «hybride» consistant en l'article 61 qui concerne la procédure applicable en cas d'inexécution d'un mandat d'arrêt. Cet article organise une audience publique permettant la comparution de témoins et la confirmation de l'acte d'accusation. S'il constitue une innovation remarquable, il n'en demeure pas moins «un monstre juridique et un succédané qui ne satisfait personne $»{ }^{47}$.

Le Statut de la Cour pénale internationale opte également clairement pour la tradition de la Common Law. De ce point de vue, la position française, favorable au jugement par contumace, a été clairement exclue par l'article 63 du Statut qui prévoit que «l'accusé assiste à son procès ». Lors des négociations, les pays de droit anglo-saxon ont en effet estimé que la faculté de prononcer une sentence in abstentia serait contraire à la crédibilité et à la légitimité de la justice internationale ${ }^{48}$. En revanche, l'article $61 \$ 2$ du Statut de Rome, s’inspirant à la fois de la solution adoptée par les juges des tribunaux ad hoc et d'une proposition de la France ${ }^{49}$, prévoit la possibilité pour la Chambre préliminaire de tenir une audience en l'absence de l'accusé en vue de confirmer les charges sur lesquelles le procureur entend fonder son

43. Nous empruntons cette démonstration à X. de Roux, «La Défense devant le Tribunal pénal pour l'ex-Yougoslavie», p. 130.

44. Voir également les remarque de C. Santulli, «La Cour pénale internationale : de l'impunité à la répression? », in La Justice pénale internationale, p. 130.

45. Sur ce point cf. également supra, I/ A.

46. X. de Roux, «La Défense devant le Tribunal pénal pour l’ex-Yougoslavie», p. 121.

47. P. Tavernier, «L'expérience des tribunaux pénaux internationaux pour l'ex-Yougoslavie et le Rwanda ", Revue internationale de la Croix-Rouge, ${ }^{\circ} 828$, 1997, p. $647-663$.

48. H. Friman, «Rights of Persons Suspected or Accused of a Crime», p. 255-261.

49. La position française a été notamment synthétisée durant les travaux préparatoires sous la forme d'un projet alternatif de Statut présenté en août 1996 (doc. A/AC.249/L.3). 
accusation. Cette possibilité permet l'enregistrement et la préservation des preuves et des témoignages ${ }^{50}$, dans l'hypothèse même où l'accusé serait introuvable. Les rédacteurs du Statut auraient pu cependant aller plus loin et admettre le jugement par contumace, dès lors qu'ils l'encadraient de précautions, sans contrevenir au respect des droits de l'accusé ${ }^{51}$.

S'agissant au contraire de la présomption d'innocence, certains auteurs ${ }^{52}$ relèvent que les statuts des tribunaux pénaux internationaux portaient $a b$ initio les stigmates du contexte de conflit armé ayant présidé à leur adoption. Il y est fait mention, il est vrai, dès l'article $1^{\text {er }}$, des «personnes présumées responsables de violations graves du droit international humanitaire». Quant bien même il ne s'agirait que d'une posture sémantique, celleci contrevient à un principe fondamental de procédure pénale $^{53}$.

C'est sur la notion de droit un procès équitable que les tribunaux internationaux de Tokyo et de Nuremberg avaient été le plus vivement critiqués. C'est à ce propos que l'on avait parlé de justice des vainqueurs. C'est la raison pour laquelle les statuts des juridictions pénales internationales contemporaines ont pris le plus grand soin à préserver les droits de la défense sur ce point.

À cet égard, le Statut de la Cour pénale internationale, en son article 55, a notamment renforcé les droits reconnus aux suspects dans le cadre d'une enquête par rapport à la procédure applicable devant les tribunaux pénaux internationaux ${ }^{54}$. L'article 67 précité, relatif aux droits de l'accusé, brosse un tableau exhaustif des standards les plus élevés en la matière ${ }^{55}$.

La pratique procédurale n'est cependant pas exactement le reflet de ces dispositions. Les droits de la défense, notamment dans le domaine de la communication des pièces et des preuves, peuvent entrer en contradiction avec les droits des États et avec la protection des victimes et des témoins. Un fort déséquilibre entre l'accusation et la défense a été régulièrement dénoncé par les avocats plaidant devant les tribunaux pénaux internationaux ${ }^{56}$.

C'est sans doute pour répondre à ces accusations que l'article 54 du Statut de la Cour pénale internationale prévoit l'obligation pour le procureur d'instruire à charge et à décharge et que l'article 57 du Statut prévoit l'institution d'une Chambre préliminaire chargée de garantir le respect des droits de la défense au stade de l'accusation.
Les critiques émises à l'encontre de l'activité des tribunaux pénaux internationaux ont rencontré d'autant plus d'écho que le fonctionnement de ces juridictions est censé annoncer le fonctionnement de la future Cour pénale internationale. C'est pourquoi la jurisprudence relative aux droits de la défense et à la notion de procès équitable a été perçue comme devant fixer le standard applicable au procès pénal international ${ }^{57}$. Sur ce point, une conclusion ambivalente s'impose : d'une part, l'activité des tribunaux pénaux internationaux a permis de fixer les règles essentielles du procès pénal international équitable, notamment au regard des droits de la défense mais, d'autre part, elle a aussi démontré les limites qui tiennent aux particularités du droit pénal international. Ainsi, dans un contexte de conflit armé, la protection des témoins et des victimes, les difficultés relatives à l'établissement des preuves et la nécessité d'obtenir la coopération des États constituent des limites aux droits de l'accusé, qu'il s'agisse de son droit à un procès équitable, de son droit à être jugé sans retard, de l'application de la détention provisoire ou encore de celle du principe non bis in idem.

S'agissant de ce dernier point, sans entrer dans un débat qui dépasserait le cadre de notre étude, il convient de noter que la future Cour, si elle se veut complémentaire des juridictions nationales - alors même que les statuts des tribunaux ad hoc instaurèrent un principe de primauté sur les juridictions nationales - dispose, au travers des article 17 à 19 relatifs à la recevabilité et malgré un article 20 intitulé "Ne bis in idem», des instruments juridiques permettant de "soustraire» un suspect, un accusé et même un condamné à une juridiction nationale.

À cet égard, la question de l'administration de la preuve devant le juge pénal international est sans doute la plus fondamentale. En effet, dans la procédure inquisitoireaccusatoire $^{58}$ applicable devant les juridictions pénales internationales, au-delà de l'impartialité et de l'indépendance du ministère public international, a priori admises, c'est au stade de l'administration et de la discussion de la preuve que se noue la notion d'équité entre l'accusation et la défense.

Devant les tribunaux pénaux internationaux, c'est avant tout le procureur qui conduit la recherche de la preuve, reposant essentiellement sur des témoignages. Or, celui-ci n'instruit qu'à charge. Il soumet ensuite acte

50. La Chambre préliminaire intervient également au stade de l'enquête pour éviter le risque de déperdition de preuves (article 56 du Statut).

51. CEDH, 12 février 1985, Colozza et Rubinat c. Italie, A, n ${ }^{\circ} 89$; CEDH, 23 novembre 1993, Poitrimol c. France, A, n ${ }^{\circ} 277-A$.

52. X. de Roux, «La Défense devant le Tribunal pénal pour l’ex-Yougoslavie», p. 128-129.

53. Il convient cependant de préciser que le respect de ce principe s'apprécie au regard de la procédure dans son ensemble: CEDH, Barbera, Messegué et Jabardo c. Espagne, 6 décembre 1988, A, nº 146.

54. United Nations, The United Nations and the Development of International Law, 1990-1999, chap. 1, p. 14 et p. 25, disponible sur internet : www.un.org (février 2003).

55. On relève ainsi le droit à être informé de la nature et de la cause de l'accusation, le droit à un interprète, le droit d'être assisté d'un conseil et le droit à la défense, le droit de ne pas s'auto-accuser, le droit à être juger sans retard excessif, la publicité des procédures. Voir F. Rubio et S. Moghaddassi, «Les garanties juridictionnelles devant la Cour pénale internationale».

56. X. de Roux, «La Défense devant le Tribunal pénal pour l'ex-Yougoslavie», p. 122-126.

57. A.-M. La Rosa, «Réflexions sur l'apport du Tribunal pénal international pour l'ex-Yougoslavie au droit un procès équitable», RGDIP, $1997-4$, p. 945.

58. G. Champy, «Inquisitoire-accusatoire devant les juridictions pénales internationales», RIDP, 1997, p. 149 et D. Mac Sweeney, «International standards of fairness, criminal procedure and the international criminal court», ibid., p. 233. 
d'accusation à la Chambre de première instance qui confirme au vu des présomptions s'il y a lieu d'engager des poursuites. La formule semble heurter le principe de la présomption d'innocence ${ }^{59}$. Puis, c'est sur la base d'indices graves et concordants tendant à montrer que le suspect aurait commis une infraction relevant de la compétence du tribunal, que le procureur pourra demander la détention provisoire. Enfin c'est sur la base de preuves convaincantes que la Chambre de première instance prononcera l'éventuelle culpabilité.

Au-delà, il est évident que le contexte de conflit armé conduit à abaisser le niveau de certitude exigé pour considérer un témoignage «crédible». Dans ce domaine, les pouvoirs des juges des tribunaux pénaux internationaux semblent à peu près illimités. Plus encore, le contexte du procès international et ses corollaires (anonymat des témoins, médiatisation du procès, obligation de collaboration des États) érode les droits de l'accusé.

Il est encore difficile, aujourd'hui, de dire si les précautions adoptées par le Statut de la future Cour pénale internationale et par son Règlement de procédure et de preuve suffiront à pallier ces difficultés et à trouver un point d'équilibre entre les droits de la défense et la nature des crimes poursuivis. La réponse sera largement dépendante de la pratique juridictionnelle et notamment de celle d'un procureur chargé d'instruire à charge et à décharge.

À cet égard, deux remarques complémentaires peuvent être ajoutées. D’une part, il apparaît que les juridictions internationales, dans leur mise en œuvre, sont frappées du sceau du système onusien et qu'elles en présentent certains travers: des accusés arrêtés depuis des années et dont les affaires demeurent au stade des exceptions préjudiciables, les lenteurs des procédures administratives internes, les moyens techniques mis en œuvre dont la lourdeur peut paralyser le fonctionnement même des juridictions, le nombre des personnels nécessaires au regard du nombre de détenus sont autant de failles qui soulèvent la question de savoir si le fonctionnement onu- sien est apte à rendre la justice. En effet, la lenteur de la justice internationale n'est pas seulement une violation des droits fondamentaux des accusés et des victimes. La lenteur de la justice la rend également incertaine ${ }^{60}$. En matière pénale, la preuve repose essentiellement sur l'interrogatoire et le contre-interrogatoire. Quelle est la fiabilité des témoignages rendus sur des faits datant de plus de dix ans? Or, la légitimité de la justice pénale est fondée sur son efficacité et sa fiabilité. Plus encore, lorsque des enjeux pécuniaires et la corruption, liés notamment au fonctionnement de la défense, viennent jeter un doute raisonnable sur la finalité de la justice internationale. Faute de pouvoir apporter des réponses satisfaisantes à ces questions, il apparaît aujourd'hui que la légitimité de la justice pénale internationale passe par l'amélioration de son fonctionnement et donc, sans doute, de sa place dans une société internationale en mutation. D'autre part, l'observation du fonctionnement des juridictions pénales internationales ad hoc démontre que, loin de marquer une véritable rupture depuis Nuremberg, la justice pénale internationale demeure une justice des vainqueurs réservée aux chefs vaincus. Cela signifie que, quelles que soient ses imperfections, cette justice plus humaine, moins expéditive et plus respectueuse des droits de la défense-mais plus coûteuse aussi-est une " politesse » des chefs vainqueurs à l'égard des élites vaincues. Dans ce cadre, le rôle des "second couteaux" - souvent des victimes parmi les bourreaux - est de servir de défouloirs aux anciennes victimes ou, au mieux, de témoins instrumentés en échange de leur accession au prétoire de cette justice modèle.

On peut souhaiter que la future Cour pénale internationale parvienne à résoudre cette ambivalence en accordant une justice toujours plus respectueuse des droits de la défense aux accusés traduits devant elle mais en servant également de référence d'efficacité de la justice aux juridictions nationales qui auront à juger de tels crimes.

59. Y. Nouvel, «La preuve devant le Tribunal pénal international pour l'ex-Yougoslavie», RGDIP, 1997-4, p. 905. 
